PROCEEDINGS OF THE

AMERICAN MATHEMATICAL SOCIETY

Volume 136, Number 10, October 2008, Pages 3513-3520

S 0002-9939(08)09290-3

Article electronically published on June 10, 2008

\title{
CUTOFF RESOLVENT ESTIMATES AND THE SEMILINEAR SCHRÖDINGER EQUATION
}

\author{
HANS CHRISTIANSON
}

(Communicated by Hart F. Smith)

\begin{abstract}
This paper shows how abstract resolvent estimates imply local smoothing for solutions to the Schrödinger equation. If the resolvent estimate has a loss when compared to the optimal, non-trapping estimate, there is a corresponding loss in regularity in the local smoothing estimate. As an application, we apply well-known techniques to obtain well-posedness results for the semi-linear Schrödinger equation.
\end{abstract}

\section{INTRODUCTION}

In this short note we show how cutoff semiclassical resolvent estimates for the Laplacian on a non-compact manifold, with spectral parameter on the real axis, lead to well-posedness results for the semilinear Schrödinger equation. Motivated by the requirements of $\mathrm{Chr} 3$ and BGT2, and the microlocal inverse estimates of Chr1, Chr2], we first prove a general theorem for a large class of resolvents. Following the recent work of Nonnenmacher-Zworski $\mathrm{NoZw}$, we apply the general theorem in the case there is a hyperbolic fractal trapped set.

Let $(M, g)$ be a Riemannian manifold of dimension $n$ without boundary, with (non-negative) Laplace-Beltrami operator $-\Delta$ acting on functions. The LaplaceBeltrami operator is an unbounded, essentially self-adjoint operator on $L^{2}(M)$ with domain $H^{2}(M)$. We assume $(M, g)$ is asymptotically Euclidean in the sense of [NoZw, $\S 3.2]$ and that the classical resolvent $\left(-\Delta-\left(\lambda^{2}+i \epsilon\right)\right)^{-1}$ obeys a limiting absorption principle as $\epsilon \rightarrow 0+, \lambda \neq 0$.

Our first result is that if we have cutoff semiclassical resolvent estimates with a sufficiently small loss, then we have weighted smoothing for the Schrödinger propagator with a loss. Let $\rho_{s}$ be a smooth, non-vanishing weight function satisfying

$$
\rho_{s}(x) \equiv\left\langle d_{g}\left(x, x_{0}\right)\right\rangle^{-s},
$$

for some fixed $x_{0}$ and $x$ outside a compact set.

Received by the editors June 29, 2007.

2000 Mathematics Subject Classification. Primary 35Q55.

This research was partially conducted during the period the author was employed by the Clay Mathematics Institute as a Liftoff Fellow.

(C)2008 American Mathematical Society Reverts to public domain 28 years from publication 
Theorem 1. Suppose for each compactly supported function $\chi \in \mathcal{C}_{c}^{\infty}(M)$ with sufficiently small support, there is $h_{0}>0$ such that the semi-classical LaplaceBeltrami operator satisfies

$$
\left\|\chi\left(-h^{2} \Delta-E\right)^{-1} \chi u\right\|_{L^{2}(M)} \leq \frac{g(h)}{h}\|u\|_{L^{2}(M)}, \quad E>0,
$$

uniformly in $0<h \leq h_{0}$, where $g(h) \geq c_{0}>0, g(h)=o\left(h^{-1}\right)$. Then for each $T>0$ and $s>1 / 2$, there is a constant $C=C_{T, s}>0$ such that

$$
\int_{0}^{T}\left\|\rho_{s} e^{i t \Delta} u_{0}\right\|_{H^{1 / 2-\eta}(M)}^{2} d t \leq C\left\|u_{0}\right\|_{L^{2}(M)}^{2}
$$

where $\eta \geq 0$ satisfies

$$
g(h) h^{2 \eta}=\mathcal{O}(1)
$$

and $\rho_{s}$ is given by (1.1).

The assumption that $(M, g)$ is asymptotically Euclidean is that there exists $R_{0}>$ 0 sufficiently large that each infinite branch of $M \backslash B\left(0, R_{0}\right)$ agrees with $\mathbb{R}^{n}$ and on each branch the semiclassical Laplacian $-h^{2} \Delta$ takes the form

$$
-\left.h^{2} \Delta\right|_{M \backslash B\left(0, R_{0}\right)}=\sum_{|\alpha| \leq 2} a_{\alpha}(x, h)\left(h D_{x}\right)^{\alpha},
$$

with $a_{\alpha}(x, h) \in \mathcal{C}_{b}^{\infty}\left(\mathbb{R}^{n}\right)$ and independent of $h$ for $|\alpha|=2$,

$$
\begin{aligned}
& \sum_{|\alpha|=2} a_{\alpha}(x, h)\left(h D_{x}\right)^{\alpha} \geq C^{-1}|\xi|^{2}, 0<C<\infty, \text { and } \\
& \sum_{|\alpha| \leq 2} a_{\alpha}(x, h)\left(h D_{x}\right)^{\alpha} \rightarrow|\xi|^{2}, \quad \text { as }|x| \rightarrow \infty \text { uniformly in } h .
\end{aligned}
$$

In order to quote the results of $\mathrm{NoZw}$ we also need the following analyticity assumption: $\exists \varepsilon>0$ such that the $a_{\alpha}(x, h)$ extend holomorphically to

$$
\left\{r \omega: \omega \in \mathbb{C}^{n}, \operatorname{dist}\left(\omega, \mathbb{S}^{n}\right)<\varepsilon, r \in \mathbb{C},|r| \geq R_{0}, \arg r \in(-\varepsilon, \varepsilon)\right\}
$$

and satisfy the same estimates in this extended region. As in $\mathbb{N o Z w}$, the analyticity assumption immediately implies

$$
\partial_{x}^{\beta}\left(\sum_{|\alpha| \leq 2} a_{\alpha}(x, h) \xi^{\alpha}-|\xi|^{2}\right)=o\left(|x|^{-|\beta|}\right)\langle\xi\rangle^{2},|x| \rightarrow \infty .
$$

Recall the free Laplacian $\left(-\Delta_{0}-\lambda^{2}\right)^{-1}$ on $\mathbb{R}^{n}$ has a holomorphic continuation from $\operatorname{Im} \lambda>0$ to $\lambda \in \mathbb{C}$ for $n \geq 3$ odd, and to the logarithmic covering space for $n$ even. This motivates the limiting absorption assumption that

$$
\lim _{\epsilon \rightarrow 0+, \lambda \neq 0} \rho_{s}\left(-\Delta-\left(\lambda^{2}+i \epsilon\right)\right)^{-1} \rho_{s}
$$

exists as a bounded operator

$$
L^{2}\left(M, d \operatorname{vol}_{g}\right) \rightarrow L^{2}\left(M, d \operatorname{vol}_{g}\right),
$$

provided $s>1 / 2$. As in the free case, we allow a possible logarithmic singularity at $\lambda=0$. 
The problem of "local smoothing" estimates for the Schrödinger equation has a long history. The sharpest results to date are those of Doi [Doi] and Burq Bur]. Doi proved if $M$ is asymptotically Euclidean, then one has the estimate

$$
\int_{0}^{T}\left\|\chi e^{i t \Delta} u_{0}\right\|_{H^{1 / 2}(M)}^{2} d t \leq C\left\|u_{0}\right\|_{L^{2}(M)}^{2}
$$

for $\chi \in \mathcal{C}_{c}^{\infty}(M)$ if and only if there are no trapped sets. Burq's paper showed if there is trapping due to the presence of several convex obstacles in $\mathbb{R}^{n}$ satisfying certain assumptions, then one has the estimate (1.5) with the $H^{1 / 2}$ norm replaced by $H^{1 / 2-\eta}$ for $\eta>0$. In Chr3, the author considered an arbitary, single trapped hyperbolic orbit. One of the goals of this paper is to use estimates obtained by Nonnenmacher-Zworski NoZw for fractal hyperbolic trapped sets to obtain similar results to $\mathrm{Chr} 3$ for the semilinear Schrödinger equation. To that end we have the following corollary to Theorem 1 .

Corollary 1.1. Assume $(M, g)$ admits a hyperbolic fractal trapped set, $K_{E}$, in the energy level $E>0$ and that the topological pressure $P_{E}(1 / 2)<0$. Then $-h^{2} \Delta-E$ satisfies (1.2) for some $E>0$ with $g(h)=C \log (1 / h)$, and for every $\eta>0, T>0$, and $s>1 / 2$, there exists a constant $C=C_{P_{E}, \eta, T, s}>0$ such that

$$
\int_{0}^{T}\left\|\rho_{s} e^{i t \Delta} u_{0}\right\|_{H^{1 / 2-\eta}(M)}^{2} d t \leq C\left\|u_{0}\right\|_{L^{2}(M)}^{2} .
$$

We remark that the assumption $P_{E}(1 / 2)<0$ implies the trapped set $K_{E}$ is filamentary or "thin" (see $\mathrm{NoZw}$ for definitions).

We consider the following semilinear Schrödinger equation problem:

$$
\left\{\begin{array}{c}
i \partial_{t} u+\Delta u=F(u) \text { on } I \times M, \\
u(0, x)=u_{0}(x),
\end{array}\right.
$$

where $I \subset \mathbb{R}$ is an interval containing 0 . Here the non-linearity $F$ satisfies

$$
F(u)=G^{\prime}\left(|u|^{2}\right) u,
$$

and $G: \mathbb{R} \rightarrow \mathbb{R}$ is at least $C^{3}$ and satisfies

$$
\left|G^{(k)}(r)\right| \leq C_{k}\langle r\rangle^{\beta-k},
$$

for some $\beta \geq \frac{1}{2}$.

In 93 we prove a family of Strichartz-type estimates which will result in the following well-posedness theorem.

Theorem 2. Suppose $(M, g)$ satisfies the assumptions of the introduction, and set

$$
\delta=\frac{4 \eta}{2 \eta+1} \geq 0 .
$$

Then for each

$$
s>\frac{n}{2}-\frac{2}{\max \{2 \beta-2,2\}}+\delta
$$

and each $u_{0} \in H^{s}(M)$ there exists $p>\max \{2 \beta-2,2\}$ and $0<T \leq 1$ such that (1.6) has a unique solution

$$
u \in C\left([-T, T] ; H^{s}(M)\right) \cap L^{p}\left([-T, T] ; L^{\infty}(M)\right) .
$$

Moreover, the map $u_{0}(x) \mapsto u(t, x) \in C\left([-T, T] ; H^{s}(M)\right)$ is Lipschitz continuous on bounded sets of $H^{s}(M)$, and if $\left\|u_{0}\right\|_{H^{s}}$ is bounded, $T$ is bounded from below. 
If, in addition, $(M, g)$ satisfies the assumptions of Corollary 1.1, $n \leq 3, \beta<3$, and $G(r) \rightarrow+\infty$ as $r \rightarrow+\infty$, then $u$ in (1.9) extends to a solution

$$
u \in C\left((-\infty, \infty) ; H^{1}(M)\right) \cap L^{p}\left((-\infty, \infty) ; L^{\infty}(M)\right) .
$$

Remark 1.2. In particular, the cubic defocusing non-linear Schrödinger equation is globally $H^{1}$-well-posed in three dimensions with a fractal trapped hyperbolic set which is sufficiently filamentary. Of course other non-linearities can be considered, but for simplicity we consider only those in this work.

\section{Proof of Theorem 1}

Since we are assuming $(-\Delta-z)^{-1}$ obeys a limiting absorption principle, we have

$$
\left\|\rho_{s}(-\Delta-(\tau-i \epsilon))^{-1} \rho_{s}\right\|_{L^{2} \rightarrow L^{2}} \leq C_{\epsilon}
$$

for $0<\epsilon_{0} \leq|\tau| \leq C$. For $|\sigma| \geq C$ for some $C>0, \sigma \in \mathbb{C}$ in a neighbourhood of the real axis, write

$$
\begin{aligned}
-\Delta-\sigma & =-\Delta-\frac{z}{h^{2}} \\
& =h^{-2}\left(-h^{2} \Delta-z\right)
\end{aligned}
$$

for

$$
z \in[E-\alpha, E+\alpha]+i\left[-c_{0} h, c_{0} h\right]
$$

Now

$$
\left(-h^{2} \Delta-z\right)
$$

is a Fredholm operator for $z$ in the specified range, and hence the "gluing" techniques from [Vod and Chr3, §2] can be used to conclude for $s>1 / 2$,

$$
\rho_{s}\left(-h^{2} \Delta-z\right)^{-1} \rho_{s}
$$

has a holomorphic extension to a slightly smaller neighbourhood in $z$, and in particular,

$$
\left\|\rho_{s}\left(-h^{2} \Delta-E\right)^{-1} \rho_{s}\right\|_{L^{2} \rightarrow L^{2}} \leq C \frac{g(h)}{h} .
$$

Rescaling, we have

$$
\left\|\rho_{s}(-\Delta-\tau)^{-1} \rho_{s}\right\|_{L^{2} \rightarrow L^{2}} \leq C \frac{g\left(\langle\tau\rangle^{1 / 2}\right)}{\langle\tau\rangle^{1 / 2}}, \tau \in \mathcal{C}_{ \pm \epsilon},
$$

where (see Figure 1)

$$
\mathcal{C}_{ \pm \epsilon}=\{\tau \in \mathbb{R}:|\tau| \geq \epsilon\} \cup\{\tau \in \mathbb{C}:|\tau|=\epsilon, \pm \operatorname{Im} \tau \geq 0\}
$$

As in Chr3 and Bur, the following lemma follows from integration by parts and interpolation, together with the condition on $\eta$, (1.4).

Lemma 2.1. With the notation and assumptions above, we have

$$
\left\|\rho_{s}(-\Delta-\tau)^{-1} \rho_{s}\right\|_{L^{2} \rightarrow H^{1}} \leq C g\left(\langle\tau\rangle^{1 / 2}\right), \quad \tau \in \mathcal{C}_{ \pm \epsilon}
$$

and for every $r \in[-1,1]$,

$$
\left\|\rho_{s}(-\Delta-\tau)^{-1} \rho_{s}\right\|_{H^{r} \rightarrow H^{1+r-\eta / 2}} \leq C, \quad \tau \in \mathcal{C}_{ \pm \epsilon} .
$$




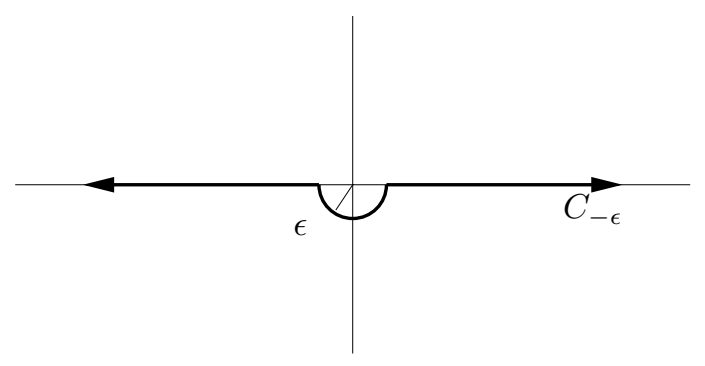

Figure 1. The curve $\mathcal{C}_{-\epsilon}$ in the complex plane.

Theorem 1 now follows from the standard " $T T^{*}$ " argument, letting $\epsilon \rightarrow 0$ in (2.1) (see [BGT2, the references cited therein, and [Chr3]).

The following corollary uses interpolation with an $H^{2}$ estimate to replace the $H^{1 / 2-\eta}$ norm on the left hand side of (1.3) with $H^{1 / 2}$, and will be of use in 93 See Chr3 for the details of the proof.

Corollary 2.2. Suppose $(M, g)$ satisfies the assumptions of Theorem 1. For each $T>0$ and $s>1 / 2$, there is a constant $C>0$ such that

$$
\int_{0}^{T}\left\|\rho_{s} e^{i t \Delta} u_{0}\right\|_{H^{1 / 2}(M)}^{2} d t \leq C\left\|u_{0}\right\|_{H^{\delta}(M)}^{2},
$$

where $\delta \geq 0$ is given by (1.7).

In particular, if $(M, g)$ satisfies the assumptions of Corollary 1.1, then for any $\delta>0$, there is $C=C_{\delta}>0$ such that (2.2) holds.

\section{StrichartZ-TyPe INEQUALities}

In this section we give several families of Strichartz-type inequalities and prove Theorem 2, The statements and proofs are mostly adaptations of similar inequalities in BGT2, so we leave out the proofs of these in the interest of space.

If we view $M \backslash U$, where $U$ is a neighbourhood of $K_{E}$, as a manifold with nontrapping geometry, we may apply the results of [HTW] or [BoTz to a solution of the Schrödinger equation away from the trapping region, resulting in perfect Strichartz estimates. For this section we need (1.3) only with a compact cutoff $\chi$ instead of with the more general weight $\rho_{s}$.

Proposition 3.1. For every $0<T \leq 1$ and each $\chi \in \mathcal{C}_{c}^{\infty}(M)$ satisfying $\chi \equiv 1$ near $U$, there is a constant $C>0$ such that

$$
\|(1-\chi) u\|_{L^{p}([0, T]) W^{s, q}(M)} \leq C\left\|u_{0}\right\|_{H^{s}(M)},
$$

where $u=e^{i t \Delta} u_{0}, s \in[0,1]$, and $(p, q), p>2$ satisfy

$$
\frac{2}{p}+\frac{n}{q}=\frac{n}{2}
$$

Remark 3.2. In the sequel, wherever unambiguous, we will write

$$
L_{T}^{p} W^{s, q}:=L^{p}([0, T]) W^{s, q}(M)
$$

and

$$
H^{s}:=H^{s}(M)
$$


Proposition 3.3. Suppose $(M, g)$ satisfies the assumptions of the Introduction, $u=e^{i t \Delta} u_{0}$, and

$$
v=\int_{0}^{t} e^{i(t-\tau) \Delta} f(\tau) d \tau
$$

Then for each $0<T \leq 1$ and $\delta \geq 0$ satisfying (1.7), we have the estimates

$$
\|u\|_{L_{T}^{p} W^{s-\delta, q}} \leq C\left\|u_{0}\right\|_{H^{s}}
$$

and

$$
\|v\|_{L_{T}^{p} W^{s-\delta, q}} \leq C\|f\|_{L_{T}^{1} H^{s}},
$$

where $s \in[0,1]$ and $(p, q), p>2$ satisfy the Euclidean scaling

$$
\frac{2}{p}+\frac{n}{q}=\frac{n}{2} .
$$

The proof uses a local WKB expansion also localized in time to the scale of inverse frequency, followed by summing over frequency bands (see Chr3 and BGT1]). The only difference here is the explicit dependence of $\delta$ on $\eta$, which is related to the growth of the function $g(h)$.

Proof of Theorem 2. The proof of Theorem 2 is a slight modification of the proof of Proposition 3.1 in BGT1, but we include it here in the interest of completeness. Fix $s$ satisfying (1.8) and choose $p>\max \{2 \beta-2,2\}$ satisfying

$$
s>\frac{n}{2}-\frac{2}{p}+\delta \geq \frac{n}{2}-\frac{1}{\max \{2 \beta-2,2\}}
$$

where $\delta \geq 0$ satisfies (1.7). Set $\sigma=s-\delta$ and

$$
Y_{T}=C\left([-T, T] ; H^{s}(M)\right) \cap L^{p}\left([-T, T] ; W^{\sigma, q}(M)\right)
$$

for

$$
\frac{2}{p}+\frac{n}{q}=\frac{n}{2}
$$

equipped with the norm

$$
\|u\|_{Y_{T}}=\max _{|t| \leq T}\|u(t)\|_{H^{s}(M)}+\|u\|_{L_{T}^{p} W^{\sigma, q}}
$$

Let $\Phi$ be the non-linear functional

$$
\Phi(u)=e^{i t \Delta} u_{0}-i \int_{0}^{t} e^{i(t-\tau) \Delta} F(u(\tau)) d \tau .
$$

If we can show that $\varphi: Y_{T} \rightarrow Y_{T}$ and is a contraction on a ball in $Y_{T}$ centered at 0 for sufficiently small $T>0$, this will prove the first assertion of the proposition, along with the Sobolev embedding

$$
W^{\sigma, q}(M) \subset L^{\infty}(M),
$$

since $\sigma>n / q$. From Proposition 3.3. we bound the $W^{\sigma}$ part of the $Y_{T}$ norm by the $H^{s}$ norm, giving

$$
\begin{aligned}
\|\Phi(u)\|_{Y_{T}} & \leq C\left(\left\|u_{0}\right\|_{H^{s}}+\int_{-T}^{T}\|F(u(\tau))\|_{H^{s}} d \tau\right) \\
& \left.\leq C\left(\left\|u_{0}\right\|_{H^{s}}+\int_{-T}^{T}\|(1+|u(\tau)|)\|_{L^{\infty}}^{2 \beta-2}\right)\|u(\tau)\|_{H^{s}} d \tau\right),
\end{aligned}
$$


where the last inequality follows by our assumptions on the structure of $F$. Applying Hölder's inequality in time with $\tilde{p}=p /(2 \beta-2)$ and $\tilde{q}$ satisfying

$$
\frac{1}{\tilde{q}}+\frac{1}{\tilde{p}}=1
$$

gives

$$
\left.\|\varphi(u)\|_{Y_{T}} \leq C\left(\left\|u_{0}\right\|_{H^{s}}+T^{\gamma}\|u\|_{L_{T}^{\infty} H^{s}}\|(1+|u|)\|_{L_{T}^{p} L^{\infty}}^{2 \beta-2}\right)\right)
$$

where $\gamma=1 / \tilde{q}>0$. Thus

$$
\|\varphi(u)\|_{Y_{T}} \leq C\left(\left\|u_{0}\right\|_{H^{s}}+T^{\gamma}\left(\|u\|_{Y_{T}}+\|u\|_{Y_{T}}^{2 \beta}\right)\right) .
$$

Similarly, we have for $u, v \in Y_{T}$,

$$
\begin{aligned}
& \|\Phi(u)-\Phi(v)\|_{Y_{T}} \\
& \left.\quad \leq C T^{\gamma}\|u-v\|_{L_{T}^{\infty} H^{s}}\|(1+|u|)\|_{L_{T}^{p} L^{\infty}}^{2 \beta-2}+\|(1+|v|)\|_{L_{T}^{p} L^{\infty}}^{2 \beta-2}\right) \\
& \left.\quad \leq C T^{\gamma}\|u-v\|_{Y_{T}}\|(1+|u|)\|_{Y_{T}}^{2 \beta-2}+\|(1+|v|)\|_{Y_{T}}^{2 \beta-2}\right),
\end{aligned}
$$

which is a contraction for sufficiently small $T$. This concludes the proof of the first assertion in the proposition.

To get the second assertion, we observe from (3.6) and the definition of $Y_{T}$, if $u$ and $v$ are two solutions to (1.6) with initial data $u_{0}$ and $u_{1}$ respectively, so

$$
\widetilde{\Phi}(v)=e^{i t \Delta} u_{1}-i \int_{0}^{t} e^{i(t-\tau) \Delta} F(v(\tau)) d \tau,
$$

we have

$$
\begin{aligned}
\max _{|t| \leq T}\|u(t)-v(t)\|_{H^{s}} & \\
= & \max _{|t| \leq T}\|\Phi(u)(t)-\widetilde{\Phi}(v)(t)\|_{H^{s}} \\
\leq C \quad & \left(\left\|u_{0}-u_{1}\right\|_{H^{s}}\right. \\
& \left.\left.\quad+T^{\gamma} \max _{|t| \leq T}\|u(t)-v(t)\|_{H^{s}}\|(1+|u|)\|_{L_{T}^{p} L^{\infty}}^{2 \beta-2}+\|(1+|v|)\|_{L_{T}^{p} L^{\infty}}^{2 \beta-2}\right)\right),
\end{aligned}
$$

which, for $T>0$ sufficiently small gives the Lipschitz continuity.

If $(M, g)$ satisfies the assumptions of Corollary 1.1, $n \leq 3, \beta<3$, and $G(r) \rightarrow$ $+\infty$ as $r \rightarrow+\infty$, we can take $s$ and $p$ satisfying $p>\max \{2 \beta-2,2\}$ and

$$
s>\frac{n}{2}-\frac{2}{p}+\delta \geq \frac{n}{2}-\frac{2}{\max \{2 \beta-2,2\}}
$$

for any $\delta>0$. Then $\sigma=s-\delta>q / n$ and the preceding argument holds. Finally, the proof of the global well-posedness now follows from the standard global wellposedness arguments from, for example, [Caz, Chapter 6]. 


\section{REFERENCES}

[BoTz] Bouclet, J-M. And Tzvetkov, N. Strichartz Estimates for Long Range Perturbations. Amer. J. Math. 129, No. 6, 2007, pp. 1565-1609. MR2369889

[Bur] Burq, N. Smoothing Effect for Schrödinger Boundary Value Problems. Duke Math. Journal. 123, No. 2, 2004, pp. 403-427. MR2066943(2006e:35026)

[BGT1] Burq, N., GÉrard, P., And Tzvetkov, N. Strichartz Inequalities and the Nonlinear Schrödinger Equation on Compact Manifolds. Amer. J. Math. 126, No. 3, 2004, pp. 569605. MR2058384 (2005h:58036)

[BGT2] Burq, N., Gérard, P., and Tzvetkov, N. On Nonlinear Schrödinger Equations in Exterior Domains. Ann. Inst. H. Poincaré Anal. Non Linéaire 21, 2004, pp. 295-318. MR 2068304 (2005g:35264)

[Caz] Cazenave, T. Semilinear Schrödinger Equations. Courant Lecture Notes in Mathematics, AMS, 2003. MR2002047(2004j:35266)

[Chr1] Christianson, H. Semiclassical Non-concentration near Hyperbolic Orbits. J. Funct. Anal. 246, 2007, no. 2, pp. 145-195. MR2321040

[Chr2] Christianson, H. Quantum Monodromy and Non-concentration Near Semi-hyperbolic Orbits, preprint. http://www.math.mit.edu/ hans/papers/qmnc.pdf

[Chr3] Christianson, H. Dispersive Estimates for Manifolds with One Trapped Orbit. To appear in Commun. PDE. http://www.math.mit.edu/ hans/papers/sm.pdf

[Doi] Dor, S.-I. Smoothing Effects of Schrödinger Evolution Groups on Riemannian Manifolds. Duke Mathematical Journal. 82, No. 3, 1996, pp. 679-706. MR1387689 (97f:58141)

[HTW] Hassell, A., TaO, T., AND Wunsch, J. Sharp Strichartz Estimates on Non-trapping Asymptotically Conic Manifolds. Amer. J. Math. 128, No. 4, 2006, pp. 963-1024. MR2251591 (2007d:58053)

[NoZw] Nonnenmacher, S. AND Zworski, M. Quantum decay rates in chaotic scattering. Sémin. Équ. Dériv. Partielles, École Polytech., Palaiseau, 2006. MR2276087 (2007i:35177)

[Vod] Vodev, G. Exponential Bounds of the Resolvent for a Class of Noncompactly Supported Perturbations of the Laplacian. Math. Res. Lett. 7 (2000), no. 2-3, pp. 287-298. MR:1764323(2001e:35134)

Department of Mathematics, Massachusetts Institute of Technology, 77 MassachuSetts Avenue, Cambridge, Massachusetts 02139

E-mail address: hans@math.mit.edu 